\title{
Comparative Evaluation of Surface Roughness of Resin- Modified Glass Ionomer and Glass Hybrid Restorative Materials Simulated by Tooth Brushing: An in-Vitro Study
}

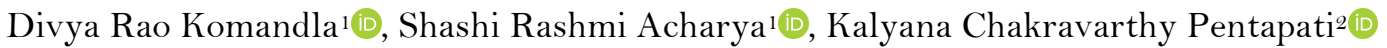

\begin{abstract}
${ }^{1}$ Department of Conservative Dentistry and Endodontics, Manipal College of Dental Sciences, Manipal, Manipal Academy of Higher Education, Manipal, India.

${ }^{2}$ Department of Public Health Dentistry, Manipal College of Dental Sciences, Manipal, Manipal Academy of Higher Education, Manipal, India.
\end{abstract}

Correspondence: Divya Rao Komandla, Department of Conservative Dentistry and Endodontics, Manipal College of Dental Sciences, Madhav Nagar, Manipal 576104, India. E-mail: divyarao369@gmail.com

Academic Editor: Catarina Ribeiro Barros de Alencar

Received: 22 December 2020 / Review: 13 May 2021 / Accepted: 10 June 2021

How to cite: Komandla DR, Acharya SR, Pentapati KC. Comparative evaluation of surface roughness of resin-modified glass ionomer and glass hybrid restorative materials simulated by tooth brushing - an in-vitro study. Pesqui Bras Odontopediatria Clín Integr. 202 1; 21 :e0259. https://doi.org/10.1590/pboci.2021.149

\begin{abstract}
Objective: To compare the effect of tooth brushing on surface roughness of Resin-Modified Glass Ionomer Cement (RMGIC; GC Gold label 2LC Light Cured Universal Restorative) and Glass Hybrid (GH; GC EQUIA SYSTEM- EQUIA Forte ${ }^{\mathrm{TM}}$ Fil and EQUIA Forte ${ }^{\mathrm{TM}}$ Coat) restorative material at 1- and 3-months interval simulated by tooth brushing. Material and Methods: RMGIC and GH material specimens (20 each) were prepared according to manufacturer instructions in $10 \mathrm{~mm} \times 2 \mathrm{~mm}$ dimensions using a mylar strip. A specially designed toothbrush simulator was used along with Oral B Pro 2 2000N powered toothbrush and Colgate Total dentifrice (Colgate-Palmolive India limited; Relative dentin abrasivity - RDA: 70 - Low abrasive) to perform brushing strokes. Specimens were subjected to surface roughness analysis before and after simulated tooth brushing at baseline, 1, and 3 months. Results: The intragroup comparison was done using repeated-measures ANOVA. Intergroup comparisons were done using an independent sample t-test and General Linear Model (ANCOVA). Surface roughness increased from baseline through 3 months in both RMGIC and GH groups. The mean surface roughness in RMGIC group was significantly higher than GH group at baseline 1 and 3-months, respectively ( $<<0.001,<0.001$, and $<0.001)$. Interaction between group and baseline surface roughness was not significant $(\mathrm{p}=0.466)$. The estimated marginal means were significantly higher in RMGIC than GH group $(p=0.008)$. Conclusion: The surface roughness of both RMGIC and GH restorative increased from baseline to 1 month and 3 months after the simulated toothbrushing protocol. GH exhibited significantly lower surface roughness than RMGIC at all the tested intervals.
\end{abstract}

Keywords: Dental Materials; Glass Ionomer Cements; Microscopy, Atomic Force. 


\section{Introduction}

Etiologically, cervical lesions are categorized as carious and non-carious lesions. The development of cervical lesions has multifactorial etiology. Brushing, diet, and occlusal or masticatory load are a few factors that can act independently at different points on the same tooth and result in the initiation and progression of cervical lesions [1].

The clinicians encounter various challenges in managing cervical lesions, viz., access to cervical lesions of posterior teeth, field control, the stress created by polymerization shrinkage on the tooth, marginal adaptation of restoration, and surface finish of the cervical restorations affecting the periodontal health. Adhesive materials like Resin-Modified Glass Ionomers (RMGIC) make a perfect choice for restoring cervical lesions. It has ion leachable glass, an organic monomer, an initiator system, and water-soluble polymeric acid. It also has better setting qualities when compared with conventional glass ionomer cement, adequate working time, which can be minimized by light curing, and swift development of initial strength, resulting in the matrix with low susceptibility for moisture contamination. Also, the translucency property is comparatively higher than conventional Glass Ionomer cement. Studies have shown RMGIC to be a suitable and successful restorative material for class $\mathrm{V}$ lesions [2-5].

The introduction of newer glass ionomer restorative materials in the market might increase the scope for success in restoring cervical lesions. One such more recent material is Glass Hybrid restorative material (EQUIA Forte system), a modification of conventional GIC with Glass hybrid technology. It has EQUIA Forte fil and EQUIA Forte coat. EQUIA Forte Fil is a rapid setting, aesthetic restorative material. The system has ultrafine glass particles that are highly reactive and dispersed within the glass hybrid fillers. As a result, they accelerate and enhance the formation of the matrix after mixing. This innovative technology improves the availability of ions and creates a strong matrix structure with superior physical properties, wear resistance, and fluoride release. EQUIA Forte Coat is a clear, light-cured resin coating that laminates EQUIA Forte Fil to polish, strengthen, protect, and contribute to the superior wear resistance of the restorative material.

Surface characteristics are one of the significant factors that govern the clinical success of any restoration. The smooth surface of restoration provides longevity, retains aesthetics, reduced plaque accumulation and surface staining [6,7]. Bollen et al. [6] reported $0.2 \mu \mathrm{m}$ as a critical threshold surface roughness value for bacterial adhesion and retention. Therefore, surface roughness tests, after simulated toothbrushing protocols, have been implied to evaluate the mechanical properties of restorative materials [8$12]$.

Existing literature on the effect of tooth brushing on surface roughness of esthetic restorative materials reported an increase in surface roughness affecting the clinical performance of cervical restorations [13-15]. In addition, various studies compared different composite resins and RMGIC for surface roughness or surface deterioration of the material, wear resistance, loss of material post brushing protocol and concluded that tooth brushing could affect the surface properties of the material [16-18].

Evaluating the deterioration capacity of restorations by simulated toothbrushing in vitro might be a surrogate parameter to assess the clinical performance of restorations. Unfortunately, information on the effect of toothbrushing on the surface roughness of glass hybrid materials is scant.

The objective of our study was to compare the effect of tooth brushing on surface roughness of resinmodified glass ionomer cement (RMGIC) and Glass hybrid (GH) restorative materials. We hypothesized that there would be significant differences in the mean surface roughness between RMGIC and GH materials. 


\section{Material and Methods}

Our study compared the micromorphological alterations on the material surface of two restorative materials (RMGIC and glass hybrid) (Table 1). Considering repeated measurements and an effect size of 0.6, with a confidence interval of $95 \%$ at $80 \%$ power, sample size estimated was 20 per group. Twenty specimens of each material were made using a lubricated stainless-steel mould of $10 \mathrm{~mm}$ x $2 \mathrm{~mm}$.

Table 1. Product name, manufacturer, composition and Lot numbers of the materials tested.

\begin{tabular}{cccc}
\hline Product Name & Manufacturer & Components & Lot No. \\
\hline $\begin{array}{c}\text { GC Gold label 2LC Light Cured } \\
\text { Universal Restorative }\end{array}$ & GC India & Powder- Fluoroaminosilicate glass (amorphous) & 1708231 \\
& & $5 \%$ Polyacrylic acid & \\
& & Liquid: Water, Polyacrylic acid, HEMA, UDMA, and & Camphorquinone \\
EQUIA SYSTEM & GC India & Fil- Fluoroaminosilicate glass, Polybasic carboxylic & 1801161 \\
(EQUIA Forte & acid, Polyacrylic acid, Water & \\
Forte ${ }^{\mathrm{TM}}$ Coat) & & Coat-Methyl methacrylate, Colloidal silica, & \\
& & Camphoroquinone, Urethane methacrylate, \\
& & Phosphoric ester monomer \\
\hline
\end{tabular}

Materials were manipulated and allowed to cure according to the manufacturer's instructions. The materials were packed in the mold and sandwiched between two mylar strips and glass slabs using constant force and within the same period. RMGIC samples were light-cured for 20 seconds from each side using LED light-curing unit (Guilin Woodpecker Medical Instrument Co., Ltd; Intensity $650 \mathrm{~mW} / \mathrm{cm}^{2}$ ). Glass hybrid specimens were coated with EQUIA Forte coat with an applicator tip after the final set of the cement and cured for 20 seconds. All the specimens were cleaned with deionized water to remove any debris present.

A specially designed toothbrush simulator along with Oral B Pro $22000 \mathrm{~N}$ powered toothbrush and Colgate Total dentifrice (Colgate-Palmolive India Ltd.; Relative dentin abrasivity (RDA:70 - Low abrasive) was used to perform brushing strokes (temperature: $37^{\circ} \mathrm{C}$; load: $250 \mathrm{gm}$ ). This toothbrush has a cross-action head with bristles angled at $16^{\circ}$. It has cleaning action by oscillation, rotation, and pulsation (48,000 movements/min). Also, the toothbrush has an inbuilt pressure sensor, which alerts the user when excessive load is applied.

Customized Brushing Simulator

A customized brushing simulator was fabricated using a commercially available powered toothbrush (Oral B pro 2 2000N powered toothbrush; Procter \& Gamble Company, Cincinnati, Ohio, USA). An acrylic handle was built to house the power toothbrush fixed to the wooden base using a nut and bolt assembly. Care was taken to ensure the bristles touch the specimens throughout the brushing protocol. Open coil spring was housed below ledge on which specimen was placed to ensure constant pressure while brushing. Specimens were placed on the acrylic stand, fixed to the wooden base (Figure 1).

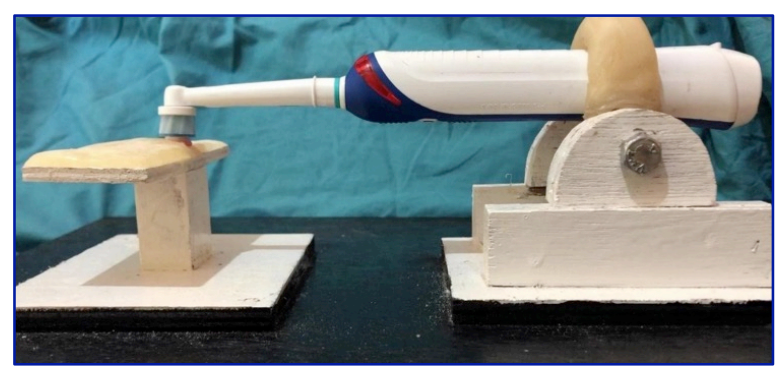

Figure 1. Customized brushing apparatus. 
Brushing Regimen

The brushing regimen involved brushing each specimen for two successive cycles $(1$ cycle $=2 \mathrm{~min}$ ) every day for three months using dentifrice slurry (Colgate Total and distilled water in 1:1 ratio). All the parameters which can affect the surface roughness of restorative materials like RDA (Relative Dentin Abrasivity) of the dentifrice used, the ratio of water/dentifrice, the nature of brush, brushing speed and the pressure applied during brushing were standardized.

The track covered by the brush was more than the diameter of the sample, ensuring the full coverage of the specimen during tooth brushing. The toothbrushing load was standardized at $250 \mathrm{~g}$. Following the brushing regimen, the specimens were rinsed and blot dried, and subjected to surface roughness analysis.

\section{Surface Roughness Analysis}

The determination of surface roughness was done by Atomic Force Microscope (Innova, Bruker). Two scans were performed for each specimen at a resolution of $10 \mu$, and average roughness values $\left(R_{a}\right)$ were recorded. In addition, digital images of the specimens at baseline, after 1 and 3 months, were obtained and analyzed for $\mathrm{R}_{\mathrm{a}}$ (Figure 2).

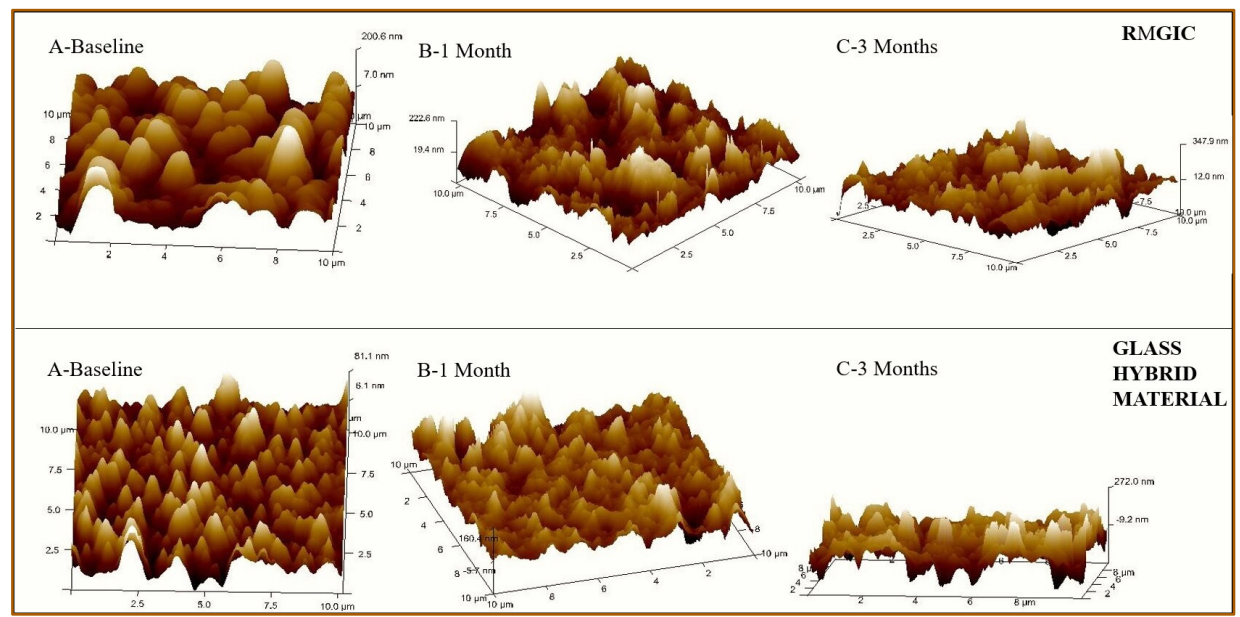

Figure 2. 3D images of one sample from each group at different intervals. Values represent the highest peak and valley values. AFM image of RMGIC specimen (A-Baseline, B-1 month and C-3 months). AFM image of Glass Hybrid specimen (A-Baselines, B-1 month and C-3 months)

\section{Ethical Clearance}

Ethical clearance for the study was obtained from the Institutional ethics committee of Kasturba Hospital and Kasturba Medical College, Manipal (716/2017).

\section{Statistical Analysis}

Statistical analysis was performed in SPSS software, version 20.0 (IBM Corp., Armonk, NY, USA). The intragroup comparison was made using repeated-measures ANOVA. A p-value of $<0.05$ was considered statistically significant. Normality was tested using Shapiro-Wilk test. Intergroup comparisons were made using independent sample t-test and General Linear Model (ANCOVA). Homogeneity of the regression was tested, checking the interaction between group and baseline roughness scores.

\section{Results}


The mean surface roughness value was significantly higher in RMGIC than the GH group at baseline, 1 and 3 months $(\mathrm{p}<0.001,<0.001$, and $<0.001)$, respectively. The intra-group analysis showed that the mean surface roughness was significantly higher at three months, followed by one month, with the least being at baseline in both RMGIC and GH groups (p<0.001 and <0.001) (Table 2$)$.

Table 2. Intra and inter-group comparisons of mean surface roughness scores.

\begin{tabular}{cccc}
\hline Evaluation & RMGIC $($ Mean \pm SD) & GH $($ Mean \pm SD) & p-value $^{\dagger}$ \\
\hline Baseline & $40.55 \pm 21.66$ & $16.25 \pm 3.21$ & $<0.001$ \\
1 Month & $88.95 \pm 37.43$ & $35.70 \pm 11.74$ & $<0.001$ \\
3 Months & $123.04 \pm 39.92$ & $51.46 \pm 12.70$ & $<0.001$ \\
p-value + & $<0.001$ & $<0.001$ & \\
& 3 Months $>$ 1 month $>$ Baseline & 3 Months $>$ 1 Month $>$ Baseline & \\
\hline +Independent sample t-test; ${ }^{\ddagger}$ Repeated measures ANOVA with post-hoc Bonferroni test.
\end{tabular}

ANCOVA was performed with baseline surface roughness as a covariate. Interaction between group and baseline surface roughness was not significant $(\mathrm{p}=0.466)$, and the estimated marginal means were significantly higher in RMGIC (115.84 \pm 7.35$)$ than in the GH group $(58.66 \pm 7.35)$ (p=0.008).

\section{Discussion}

Surface characteristics are one of the significant factors that govern the clinical success of any restoration. The smooth surface of restoration provides longevity, retains aesthetics, reduces plaque accumulation and surface staining $[6,7]$.

Substantial variations among the materials with respect to wear and surface roughness were reported because of a combination of factors [19,20]. One of these factors is the nature and property of the matrix, which is produced by an acid-base cross-linking interaction of the polyacrylic acid and metal ions (conventional GIC) by an interpenetrating network of polymer amalgamating the acid-base cross-linking reaction with the cross-linking polymerization of the monomer system, or by an additive action of polymers (resin-modified glass-ionomer cement). The proportion and dimensions of glass in organic particles were also shown to affect the wear and surface roughness of materials. In addition, Wilson and Kent identified the distinct phases in the setting reaction formed due to inability of hydrogel salt from ionomers and poly-HEMA to interpenetrate each other, thus resulting in distinct phases, which is not anticipated for the desired properties of the material [21]. However, the interpenetration of the phases is avoided in EQUIA Forte material as it is a glass hybrid material with no resin component, which might have contributed to the improved surface roughness property compared to resin-modified GIC.

Finishing and polishing protocols also influence the surface roughness property of restorative materials. However, a mylar strip was used in the present study to obtain a smooth surface as recommended in the literature [22].

Before toothbrush abrasion, the mean Ra-values of RMGIC were significantly more than the GH group. Probable reasons might be due to the application of surface coat (EQUIA Forte Coat) in the GH group. A protective coating either with light polymerized low viscosity unfilled resin adhesives or G-coat plus is compulsory for all the glass ionomer materials to enhance the wear resistance [23,24]. Conversely, some studies reported no difference in surface roughness values in coated and uncoated specimens [14]. Also, studies have reported that the application of protective coats on the glass ionomer restorations severely impedes the 
fluoride release from the restorations. Hence it was advised that it is better not to coat the GIC materials when the fluoride release property is more important than other properties. Supposedly, the resin coat (EQUIA Forte Coat) infiltrates the surface of restorative material, covering all the gaps, fissures, and porosities of GH material (EQUIA Forte fil), thus enhancing the surface smoothness.

GIC materials are diverse materials with biphasic nature and consist of unreacted glass particles surrounded by a poly-salt resin matrix. The size, shape, concentration, and composition of the glass ionomer materials' glass particles critically affect the surface roughness and the resistance to wear [25]. In the present study, this may be the possible explanation as to why the two tested GIC materials exhibited differences in their properties. During toothbrushing, the soft matrix phases were favorably removed, leaving the harder, non-reacted glass particles to bulge out from the surface and contribute to the increased surface roughness of the restorative. The hybrid-resin-modified glass ionomers showed comparable performance to traditional resin-based composite materials regarding resistance to toothbrush wear. No relationship between wear resistance and filler content has been found [16]. However, studies reported the filler particle size in the matrix having a pronounced effect on the roughness property of the material.

Bollen et al. [6] reported $0.2 \mu \mathrm{m}$ as a critical threshold value for bacterial adhesion, and retention and value of $0.25-0.5 \mu \mathrm{m}$ roughness can be sensed by the patient's tongue. In the present study, however, the increased values post brushing protocol were below the threshold limits. Although the values obtained were well within the threshold limits, there was a significant increase in the values from baseline to 3 months, which is expected to increase if the tooth brushing protocol is continued. Also, if the values increased at a similar rate as in the present study, it would exceed the critical threshold limit of surface roughness leading to rough surface restorations in the long run.

In our study, surface roughness values were significantly higher in RMGIC than in the GH group at all the tested time points. A probable reason for the obtained result might be the difference in the setting mechanism of both the materials or the application of coat on glass hybrid material.

Uppal et al. [26] considered re-polishing the materials after tooth brushing and concluded that polishing protocols could be adopted to restore a smooth surface on esthetic restorative materials following tooth brushing. However, the effect of re-polishing on resin-modified glass ionomers and glass hybrid restorative materials was not evaluated.

Few limitations were seen in our study as only one type of toothbrush and dentifrice were used to evaluate the surface roughness. Increased surface roughness may not wholly predict the clinical performance of restorative materials. Factors like stresses acting on cervical restorations, the impact of food and beverages, marginal microleakage and flexural strength could have implications on the clinical performance of the restorations, were not evaluated in this study [27]. Future research should consider factors like bacterial adhesion, plaque retention, staining potential, and the surface roughness to establish correlation and clinical performance of these materials.

\section{Conclusion}

Tooth brushing increased the surface roughness values of both RMGIC and GH from baseline to 3 months. However, GH showed significantly lower surface roughness than RMGIC. 


\section{Authors' Contributions}

$\begin{array}{lll}\text { DRK (D) https://orcid.org/0000-0002-4038-0226 } & \begin{array}{l}\text { Conceptualization, Methodology, Formal Analysis, Investigation, Data Curation, Writing - } \\ \text { Original Draft and Writing - Review and Editing. } \\ \text { Conceptualization, Methodology, Validation, Writing - Original Draft, Writing - Review and } \\ \text { Editing and Supervision. }\end{array} \\ \text { SRA } & \text { (D) https://orcid.org/o0o0-0001-8906-6353 } & \begin{array}{l}\text { Validation, Resources, Writing - Review and Editing, Visualization and Supervision. } \\ \text { KCP }\end{array} \\ \text { All authors declare that they contributed to critical review of intellectual content and approval of the final version to be published. }\end{array}$

\section{Financial Support}

None.

\section{Conflict of Interest}

The authors declare no conflicts of interest.

\section{Data Availability}

The data used to support the findings of this study can be made available upon request to the corresponding author.

\section{References}

[1] Bader JD, McClure F, Scurria MS, Shugars DA, Heymann HO. Case-control study of non-carious cervical lesions. Community Dent Oral Epidemiol 1996; 24(4):286-91. https://doi.org/10.1111/j.1600-0528.1996.tbo0861.x

[2] Maneenut C, Tyas MJ. Clinical evaluation of resin-modified glass-ionomer restorative cements in cervical "abrasion" lesions: one-year results. Quintessence Int 1995; 26(10):739-43.

[3] Neo J, Chew CL, Yap A, Sidhu S. Clinical evaluation of tooth-colored materials in cervical lesions. Am J Dent 1996; $9(1): 15-8$.

[4] Loguercio AD, Reis A, Barbosa AN, Roulet JF. Five-year double-blind randomized clinical evaluation of a resinmodified glass ionomer and a polyacid-modified resin in noncarious cervical lesions. J Adhes Dent 2003; 5(4):323-32.

[5] van Dijken JW V, Pallesen U. Long-term dentin retention of etch-and-rinse and self-etch adhesives and a resinmodified glass ionomer cement in non-carious cervical lesions. Dent Mater 2008; 24(7):915-22. https://doi.org/10.1016/j.dental.2007.11.008

[6] Bollen CM, Lambrechts P, Quirynen M. Comparison of surface roughness of oral hard materials to the threshold surface roughness for bacterial plaque retention: a review of the literature. Dent Mater 1997; 13(4):258-69. https://doi.org/10.1016/s0109-5641(97)80038-3

[7] Lu H, Roeder LB, Lei L, Powers JM. Effect of surface roughness on stain resistance of dental resin composites. J Esthet Restor Dent 2005; 17(2):102-8; discussion 109. https://doi.org/10.1111/j.1708-8240.2005.tbo0094.x

[8] Cunha LG, Alonso RCB, Santos PH Dos, Sinhoreti MAC. Comparative study of the surface roughness of Ormocerbased and conventional composites. J Appl Oral Sci 2003; $11(4): 348-53$.

https://doi.org/10.1590/s1678-77572003000400014

[9] Garcia FCP, Wang L, D’Alpino PHP, Souza JB de, Araújo PA De, Mondelli RF de L. Evaluation of the roughness and mass loss of the flowable composites after simulated toothbrushing abrasion. Braz Oral Res 2004; 18(2):156-61. https://doi.org/10.1590/S1806-83242004000200012

[10] Wang L, Garcia FCP, Amarante de Araujo P, Franco EB, Mondelli RFL. Wear resistance of packable resin composites after simulated toothbrushing test. J Esthet Restor Dent 2004; 16(5):303-5. https://doi.org/10.1111/j.1708-8240.2004.tb00058.x

[11] Prakki A, Cilli R, Mondelli RFL, Kalachandra S. In vitro wear, surface roughness and hardness of propanalcontaining and diacetyl-containing novel composites and copolymers based on bis-GMA analogs. Dent Mater 2008; 24(3):410-7. https://doi.org/10.1016/J.DENTAL.2007.06.011

[12] Cilli R, de Mattos MCR, Honorio HM, Rios D, de Araujo PA, Prakki A. The role of surface sealants in the roughness of composites after a simulated toothbrushing test. J Dent 2009; 37(12):970-7. https://doi.org/10.1016/j.jdent.2009.08.002

[13] Dudás C, Forgó Z, Kerekes-máthé B. Surface Roughness changes of different restoration materials after tooth brushing simulation using different toothpastes. J Interdiscip Med 2017; 2:21-4. https://doi.org/10.1515/jim-2017-0012

[14] Pacifici E, Bossù M, Giovannetti A, La Torre G, Guerra F, Polimeni A. Surface roughness of glass ionomer cements indicated for uncooperative patients according to surface protection treatment. Ann Stomatol 2014; 4(3-4):250-8.

[15] Bezgin T, Ozer L, Tulga Oz F, Ozkan P. Effect of toothbrushing on color changes of esthetic restorative materials. J Esthet Restor Dent 2015; 27(Suppl 1):S65-73. https://doi.org/10.1111/jerd.12136

[16] Frazier KB, Rueggeberg FA, Mettenburg DJ. Comparison of wear-resistance of Class V restorative materials. J Esthet Dent 1998; 10(6):309-14. https://doi.org/10.1111/j.1708-8240.1998.tbo0509.x 
[17] Franco EB, Benetti AR, Ishikiriama SK, Santiago SL, Lauris JRP, Jorge MFF, et al. 5-year clinical performance of resin composite versus resin modified glass ionomer restorative system in non-carious cervical lesions. Oper Dent 2006; 31(4):403-8. https://doi.org/10.2341/05-87

[18] Brackett MG, Dib A, Brackett WW, Estrada BE, Reyes AA. One-year clinical performance of a resin-modified glass ionomer and a resin composite restorative material in unprepared Class V restorations. Oper Dent 2002; 27(2):112-6.

[19] Heath JR, Wilson HJ. Abrasion of restorative materials by toothpaste. J Oral Rehabil 1976; 3(2):121-38. https://doi.org/10.1111/j.1365-2842.1976.tboo936.x

[20] Sidhu SK, Sherriff M, Watson TF. In vivo changes in roughness of resin-modified glass ionomer materials. Dent Mater 1997; 13(3):208-13. https://doi.org/10.1016/S0109-5641(97)80028-0

[21] Wilson AD, Kent BE. A new translucent cement for dentistry. The glass ionomer cement. Br Dent J 1972; 132(4):133-5. https://doi.org/10.1038/sj.bdj.4802810

[22] Mallya PL, Acharya S, Ballal V, Kundabala M, Thomas M. Profilometric study to compare the effectiveness of various finishing and polishing techniques on different restorative glass ionomer cements. J Interdiscip. Dent 2013; 3(2):86-90. https://doi.org/10.4103/2229-5194.126867

[23] Lohbauer U, Kramer N, Siedschlag G, Schubert EW, Lauerer B, Muller FA, et al. Strength and wear resistance of a dental glass-ionomer cement with a novel nanofilled resin coating. Am J Dent 2011; 24(2):124-8.

[24] Hajilou S, Zajkani E, Naghili A. Effect of a resin coating material on the microleakage of class v restorations with or without post-operative bleaching. Pesqui Bras Odontopediatria Clin Integr 2020; 20:1-8. https://doi.org/10.1590/pboci.2020.121

[25] Dionysopoulos D, Dionyssopoulos P. Surface finish produced on 5 aesthetic restorative materials by new polishing systems. Balk J Stomatol 2012; 16(1):27-33.

[26] Uppal M, Ganesh A, Balagopal S, Kaur G. Profilometric analysis of two composite resins' surface repolished after tooth brush abrasion with three polishing systems. J Conserv Dent 2013; 16(4):309. https://doi.org/10.4103/0972-0707.114356

[27] Moshaverinia M, Navas A, Jahedmanesh N, Shah KC, Moshaverinia A, Ansari S. Comparative evaluation of the physical properties of a reinforced glass ionomer dental restorative material. J Prosthet Dent 2019; 122(2):154-9. https://doi.org/10.1016/j.prosdent.2019.03.012 